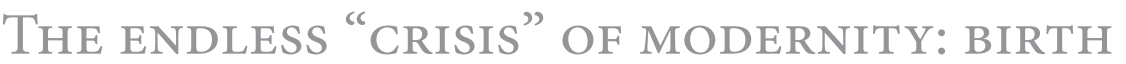

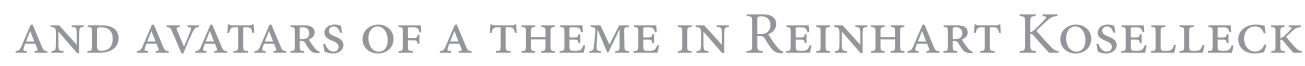 La « crise » sans fin de la modernité : naissance et avatars d'un thème chez Reinhart Koselleck
}

\section{Alexandre Escudier}

\section{CEVIPOF, Fondation Nationale des Sciences Politiques, Paris}

alexandre.escudier@sciencespo.fr - https://orcid.org/0000-0001-6107-3578

\section{Fecha recepción: 29.05.2020 / Fecha aceptación: 14.09.2020}

\section{Résumé}

La question de la «crise » a occupé Reinhart Koselleck sa vie durant. A partir de sa thèse de doctorat, Kritik und Krise (1954), elle a très tôt déterminé sa perception du monde moderne - des guerres civiles confessionnelles du $\mathrm{XVI}^{\mathrm{e}}$ siècle jusqu'au long cycle révolutionnaire de la fin du XVIII ${ }^{e}$ siècle. Dans sa première partie, l'article propose une analyse critique de Kritik und Krise. Sa conclusion intermédiaire est double. D’une part, il montre l'existence d'une triple surcharge philosophique de la démonstration koselleckienne (surchage schmittienne, cochinienne et löwithienne). D'autre part, il relève les principaux forçages interprétatifs et empiriques sur lesquels repose la thèse générale du livre. Dans sa seconde partie, l'article examine la manière dont cette première analyse de la «crise »

\begin{abstract}
The question of the "crisis" occupied Reinhart Koselleck throughout his life. From his doctoral thesis 'Kritik und Krise' (1954) onwards, this very question determined his perception of the modern world - from the religious civil wars of the 16th century to the long revolutionary cycle of the late 18 th century. In the first part, the article delivers a critical analyse of 'Kritik und Krise'. His intermediate conclusion is twofold. On the one hand, it shows the existence of a triple philosophical overload of the Koselleckian argument (listed here as schmittian, cochinian and löwithian overload). On the other hand, it reveals the main interpretative and empirical thrust that keeps afloat the general argument of the book. In the second part, the article examines the way in which this first analysis
\end{abstract}


par Koselleck évolue par la suite dans son œuvre à travers les thématiques de la "Sattelzeit ", de la " temporalisation " (Verzeitlichung) et de l'« accélération " (Beschleunigung). L'article défend au final une double thèse : d'une part, l'idée que le premier Koselleck de Kritik und Krise fait moins œuvre d'historien que de critique généalogique de la modernité totalitaire; d'autre part, l'idée que la sur-accentuation ultérieure de «l'accélération » (en lieu et place de la « critique » crisogène moderne) a permis à Koselleck d'anticiper certains questionnements de l'anthropocène alors même que ses interrogations initiales relevaient d'une ontologie politique néo-hobbésienne peu à même de penser le tournant démocratique moderne.

\section{Mots-clés}

Reinhart Koselleck, absolutisme, crise, Lumières, Sattelzeit, temporalisation, accélération of the "crisis" by Koselleck subsequently evolves in his work through the themes of "Sattelzeit", "temporalization" (Verzeitlichung) and "acceleration" (Beschleunigung). The article ultimately defends a double thesis: (a) the idea that 'Kritik and Krise' is less the work of an historian than a genealogical criticism of totalitarian modernity, but also (b) the idea that the subsequent over-emphasis of "acceleration" (instead of the modern crisogenic "criticism") allowed Koselleck to anticipate some questions regarding the Anthropocene era, despite the obvious fact that his initial inquiry was part of a neo-Hobbesian political ontology that was unable to provide a satisfying account of the modern democratic turning point.

\section{Keywords}

Reinhart Koselleck, absolutism, crisis, Enlightenment, saddle period (Sattelzeit), temporalization, acceleration 
L'ceuvre de Reinhart Koselleck est vaste et complexe. Elle touche à l'histoire des idéologies et des langages politiques modernes, à l' histoire sociale et institutionnelle de l' Etat prussien mais aussi à l'iconographie politique comparée, à l'histoire des monuments aux morts et à la théorie de l'histoire. Un si large spectre induit nécessairement une évolution interne au fil du temps, voire une certaine hétérogénéité. Certains thèmes transversaux naissent pourtant très tôt, comme celui de la " crise " dans la modernité ou de la modernité comme "crise ", et se maintiennent jusqu'à la fin, moyennant quelques transformations de fond. C'est ce qu' il s'agit d'examiner ci-après en repartant du premier travail académique de Koselleck intitulé Kritik und Krise (1954/1959)1.

Dans un premier temps, je m'efforcerai de dégager les articulations principales de la thèse défendue dans KuK quant à la dialectique de l' Etat absolutiste. Je marquerai la nature et les limites de sa méthode comme de ses sources ; j’ indiquerai également la surdétermination philosophique de sa grille d'analyse. Dans un second temps, je donnerai une interprétation d'ensemble de la manière dont, par-delà KuK, Koselleck a continué à argumenter la question de la crise à

1. Une mise au point d'ordre philologique s'impose d'entrée de jeu car il existe plusieurs versions de Kritik und Krise, avec notamment des sous-titres dissemblables. Une première variante est constituée par la version dactylographiée ayant donné lieu à la soutenance de thèse sous le titre: Kritik und Krise : Eine Untersuchung der politischen Funktion des dualistischen Weltbildes im 18. Jahrhundert, sous la direction de Johannes Kühn, université de Heidelberg, 20 novembre 1954, IX-156 p. Une seconde version a paru cinq ans plus tard sous l'intitulé: Kritik und Krise: eine Studie zur Pathogenese der bürgerlichen Welt, Fribourg/ Brisgau-Munich, 1959, X-229 p. ; elle a été rééditée en 1969. Une troisième version modifiée, comportant des compléments dans les notes (en forme de sémantique historique sur de grandes notions alors en jeu) est publiée avec le même sous-titre chez Suhrkamp à Francfort/Main, en 1973. Il s'agit de l'édition définitive. Elle a été régulièrement réimprimée de 1976 à 2011. La traduction française paraît en 1979 sous l'intitulé Le règne de la critique (Paris, Minuit); elle a été réalisée par Hans Hildenbrand à partir de l'édition de 1959. Elle est par conséquent incomplète, notamment quant aux micro-études «begriffsgeschichtlich» ajoutées dans les notes par Koselleck dans l'édition Suhrkamp de 1973. Je citerai ci-après en abrégé « KuK » pour l'édition Suhrkamp définitive de 1973 et «C» pour la traduction française de 1979. La reconstruction critique que je propose restera donc incomplète tant que n'auront pas été conduits un repérage et une analyse systématiques des modifications opérées par Koselleck entre la version de 1954, 1959 et 1973. Dans le cadre du présent article, je me contenterai simplement de signaler les passages de 1959 introuvables dans la version de 1973 sur les questions précises qui me retiennent ici, et non pour l'ensemble du texte de KuK. 
travers les thématiques de la «Sattelzeit », de la «temporalisation» (Verzeitlichung) et de l" « accélération" (Beschleunigung). Cela me permettra de marquer les continuités et discontinuités de ses axiomes interprétatifs. Mon propos relève de ce qu'on pourrait appeler un «scepticisme endetté»; entendons par là qu' il me semble à la fois nécessaire de critiquer certaines thèses de Koselleck et de continuer d'en reconnaître la productivité heuristique comme questionnement fondamental sur la modernité. Il y a en effet plusieurs manières non hagiographiques d' hériter d'une pensée, et la critique n'est pas la moins infidèle ni la moins reconnaissante des attitudes. Je précise d'emblée qu' il ne s'agit nullement pour moi ici de procéder par reductio ad schmittum intégrale, alors même qu'une véritable surcharge interprétative schmittienne me semble tout à fait repérable, et intenable, dans le propos initial de $\mathrm{KuK}^{2}$.

\section{Reconstruction critique des thèses centrales de Kritik und Krise}

KuK est rarement lu de près. On prend rarement soin de dégager les différentes thèses qui s'y trouvent patiemment enchaînées en forme de " dialectique des Lumières " (C135$\mathrm{K} 135)^{3}$. On se donne encore moins la peine d'examiner comment l'argumentation générale est concrètement construite, à partir de quels types de sources, selon quelle méthode et quels présupposés interprétatifs. KuK fonctionne ainsi le plus souvent comme une référence obligée. On le cite sans véritable distance ni discussion du détail, à l'image de l'œuvre entière de Koselleck qui fonctionne comme une sorte d'attracteur magnétique auquel il est

2. Il convient de strictement distinguer ici entre présupposés philosophiques (quant aux critères de viabilité de tout ordre politique) et préjugés idéologiques (i.e. les affects anti-libéraux de la génération de Schmitt, Brunner et Conze). Foncièrement hobbésien, Koselleck ne se départit pas d'un certain nombre de présupposés philosophiques, qu' il partage en partie avec Carl Schmitt. Il en va tout autrement des préjugés idéologiques, aussi bien pour des questions de tempérament personnel que d'expérience générationnelle. C’est pourquoi aucune reductio ad schmittum intégrale de Koselleck n'est à mon sens tenable. Ma critique, ici ou là sévère, s' inscrit dans ces bornes précises, et on ne lui fera pas dire ce qu'elle ne dit à aucun moment, sauf pour un lecteur prévenu et pressé, autant dire insignifiant. Le jugement d' Ivan Nagel demeure sur ce point définitif: «Bei sorgfältiger Lektüre von Kritik und Krise entdeckt man freilich, wie präzis sich Koselleck von Carl Schmitt absetzt: Er denkt den Staat von Hobbes aus, nicht auf Donoso Cortès hin. Eine als Anthropologie verkleidete Theologie, die gegen die Erbsünde des stets bösen Menschen total den Staat (oder : den totalen Staat) ermächtigt - diese düstere Theologie von Menschenargwohn und Menschheitsfurcht blieb dem Aufklärer über Aufklärung, der Koselleck war und ist, nicht nur im Gedanken, sondern dank Konstitution und Temperament anrüchig und fremd », I. Nagel, «Der Kritiker der Krise » (2004), in Stefan Weinfurter (ed.), Reinhart Koselleck (1923-2006) : Reden zum 50. Jahrestag seiner Promotion in Heidelberg, Heidelberg, 2006, 22-31, 27. Sur la reductio ad schmittum, cf. Koselleck lui-même in Id., " Dankrede » (23.11.2004), in S. Weinfurter (ed.), Reinhart Koselleck... op. cit., 33-60, 55.

3. Dialektik der Aufklärung aurait d'ailleurs dû être le titre du livre si Adorno et Horkheimer ne l'avaient déjà utilisé avant Koselleck, cf. «Dankrede» (23.11.2004), in S. Weinfurter (ed.), Reinhart Koselleck... op. cit., 34. 
difficile de se soustraire, de sorte à penser à la fois avec et contre son auteur, là où le discernement des plus tard venus le requiert.

Koselleck y énonce pourtant plusieurs thèses intermédiaires qui, articulées les unes aux autres, lui permettent de porter un diagnostic général que l’on pourrait résumer ainsi : l'Etat absolutiste a permis l'épanouissement de la « société » grâce à la neutralisation des guerres civiles confessionnelles en interne et l'équilibre des puissances monarchiques en externe, mais il a fini par succomber à la remoralisation dualisante, agonistique, du politique par la critique des philosophes d'abord, des cercles urbains de discussion ensuite (loges maçonniques incluses), et l'inflation des philosophies de l'histoire à visée utopique enfin - toutes choses qui nous auraient fait durablement entrer dans le cycle totalitaire moderne des révolutions. Examinons comment cette thèse générale est élaborée.

\subsection{La structure politique de l'absolutisme}

Le premier moment de l'analyse porte sur la «structure politique de l'absolutisme » (chap. 1) en tant qu'elle prépare le terrain à une société soucieuse de progrès moral dans l'histoire et par là même capable de critique fondamentale de l'ordre étatique. D'obédience clairement schmittienne, le motif est développé à deux niveaux dissemblables et complémentaires : la politique interne (Hobbes) et la politique externe (Vattel) des Etats absolutistes.

Reposant à y bien regarder sur un corpus de sources littéraires et philosophiques fort limité (Barclay, Agrippa d'Aubigné, Hobbes, Spinoza, Vattel), le premier niveau de l'analyse consiste à montrer comment l'Etat absolutiste a privatisé l'espace intérieur de la conscience morale en neutralisant les différends confessionnels qui faisaient rage depuis la Réforme dans l'espace public. En mettant un terme à la guerre civile, en se posant comme le garant de la stabilité non stasique de l'ordre politique, l'Etat absolutiste a non seulement subordonné la morale aux fins premières du politique (la paix et la sécurité), mais il l'a surtout détachée de sa matrice religieuse. La conscience religieuse est désormais sommée de se couper des affaires du siècle. «Pour survivre, le sujet doit cacher sa conscience» (C17-K15). Il en résulte en chaque individu deux parts distinctes, non médiatisées: le «sujet» de l'Etat absolutiste d'un côté et le «for intérieur» de la conscience morale de l'autre. Ce faisant, le sujet de l'Etat est délié de toute responsabilité politique, et le for intérieur devient le foyer à partir duquel quelque chose comme une critique morale de l'ordre existant peut en réaction se dégager.

Le propos de Koselleck est bien de nature «dialectique» au sens où il s'agit de montrer que l'ordre instauré par l'Etat absolutiste afin de résoudre un premier problème - les guerres de religion, et la guerre civile en général - en induit structurellement un autre, qui éclatera finalement à la fin du cycle absolutiste avec le début de l'ère révolutionnaire transatlantique $(1776,1789)$. En se posant, l'Etat absolutiste instaure les conditions de possibilité structurelles de sa critique comme de sa ruine. L'ordre positif se pose en même temps qu'il pose sa possible négation.

L'Etat a créé un ordre nouveau, dont il a été ensuite la victime. Dès le départ, le for intérieur de la morale, concédé par l'Etat et réservé à l' homme en tant qu' 'homme', était un foyer de troubles

Revista de historiografía 34, 2020, pp. 53-72 
propres au système absolutiste. L'instance de la conscience était le reste non surmonté de l'état naturel, qui survivait dans l'Etat arrivé à sa perfection. La neutralisation de la conscience par la politique favorise la sécularisation de la morale (C32-K30).

Toujours incandescent sous la cendre, ce foyer de braise sera réactivé au XVIII siècle lorsque la jonction pourra s'opérer entre de nouvelles couches sociales, des formes inédites de sociabilité et les puissances critiques de la philosophie. C'est en ce sens que «l'esprit bourgeois a recueilli la succession de la religiosité théologique» (C33-K31). Mais il est clair ici que Koselleck travaille avec des catégories historiographiques amples et vagues, avec des sortes de quasi-sujets collectifs non objectivables empiriquement («esprit bourgeois», «religiosité théologique», «l' homme», le « for intérieur », etc.). Il est également manifeste que Hobbes sert de fil conducteur à sa reconstruction d'ensemble, comme si un corpus philosophique sans efficace historique propre pouvait permettre de cartographier l'effectivité socio-historique advenue $e^{4}$.

A un second niveau (la politique externe de l'Etat absolutiste), Koselleck s'appuie essentiellement sur Le Droit des gens de Vattel ${ }^{5}$. Il s'emploie à montrer que l'état de nature belliqueux toujours possible entre les Etats a été jugulé par le «droit des gens» et l'équilibre des puissances en Europe depuis les traités de Westphalie (1648) et surtout la paix d'Utrecht (1713), qui a durablement consacré la fin de l'hyperpuissance française. On reconnaît ici bien sûr le thème du « jus publicum europaeum » chez Carl Schmitt dans Le Nomos de la terre $^{6}$. Ceci étant, Koselleck n'entre pas en matière sur un aspect important de la proposition schmittienne, à savoir que l'équilibre interne du système des Etats européens s'est soldé par une concurrence maritime et coloniale intense (des " prises de terre ») dont la caractéristique essentielle était de reconduire ailleurs, et aux dépens d'autres territoires et populations, l'affrontement guerrier ouvert qui avait été pour un temps contenu en Europe. Sous un mode thalassocratique et colonial inédit, la politique externe de l'Etat absolutiste pérennise l'agonistique des puissances et ne consiste donc pas seulement, comme en interne, à subordonner la morale à la politique de sorte à circonscrire la violence illimitée de la guerre juste.

Quoiqu'il en soit, la conclusion de Koselleck ne retient à ce stade que ceci :

Le fait fondamental du XVIII ${ }^{e}$ siècle est donc l'épanouissement du monde moral dû à la stabilité politique préalablement assurée. C’est seulement par la neutralisation politique des conflits religieux et par la limitation des guerres à des guerres purement étrangères qu'a été dégagé l'espace social où la nouvelle élite pouvait s'épanouir. En comparaison avec le passé, le citoyen se sentait en sécurité au sein de cet ordre. (...) Avec comme toile de fond la sécurité régnante, la foi qu'avait la philosophie de l' histoire dans le progrès moral de l'homme civil a acquis son évidence historique. Le progrès moral est un produit de la stabilité politique (C40-K38).

4. On comparera par exemple le caractère massif de KuK à la finesse des analyses de F. Cosandey et R. Descimon, L'absolutisme en France, Paris, 2002.

5. E. de Vattel, Le Droit des gens: Principes de la loi naturelle, appliqués à la conduite et aux affaires des Nations et des Souverains, 2 vol., Londres/ Neuchâtel, 1758.

6. C. Schmitt, Der Nomos der Erde im Völkerrecht des Jus Publicum Europaeum, Cologne, Greven, 1950. 
Contenue en Europe même, la guerre donnait l'illusion d'avoir tout à fait disparu? Cette situation, propice à la croyance en un possible progrès moral, était à la fois le produit structurel de l'ordre absolutiste et le grand leurre anti-politique moderne quant aux conditions de possibilité pérennes de toute paix et de tout ordre politique (interne comme externe): l'Etat.

\subsection{Situation philosophique et sociologique des Lumières}

Le second moment de l'analyse (chap. 2) consiste à montrer comment, depuis la fin du XVII siècle, la société civile - cette créature induite par l'ordre pacifié de l'Etat absolutiste - s'emploie à remoraliser l'ordre politique à partir du for intérieur individuel, de la critique philosophique et de nouvelles pratiques urbaines de sociabilité. Les Lumières philosophiques, dès la fin du XVII ${ }^{e}$ siècle, équivaudraient selon Koselleck à une dilatation vers l'espace public du for intérieur privé que l'Etat absolutiste avait relégué hors de la sphère politique et de sa structuration éthico-religieuse (C43-K41). Le mouvement du politique va alors du dedans vers le dehors, du for intérieur vers l'Etat en passant par la «société» qui, collection de consciences morales extériorisées, finira par se dresser en face du pouvoir absolutiste afin de le réinstituer intégralement à partir d'elle seule de sorte à pouvoir dire en substance : non pas seulement L'Etat, c'est nous, mais L'Etat, c'est nous en tant que Critique morale illimitée de droit.

Koselleck repart pour ce faire de John Locke, mais le fait remarquable est qu' il fait totalement l'impasse sur les Two Treatises of Government pour ne retenir qu' un seul passage, fort bref, de l'Essay on human understanding (II, 28, $\$ 7-10$ ). C'est dans ces quelques pages que Locke avance la triade «divine law», « civil law», «philosophical law» (appelée également «law of opinion or reputation »).

Koselleck a de ce texte une lecture maximaliste. Il y voit essentiellement trois choses : 1) un réarmement normatif de la « loi divine » contre l'ordre supra-confessionnel de l'Etat argumenté par Hobbes pour mettre fin au quasi état de nature de la guerre civile ; 2) une autonomie normative attribuée aux jugements moraux formulés depuis la « loi philosophique » et ses instances dialogiques dans la société (notamment les clubs) ; 3) summa summarum, autant de sources de normativité orthogonales aux « lois civiles » du Léviathan. La conclusion de Koselleck est radicale:

En interprétant la loi philosophique, Locke a investi politiquement le for intérieur de la conscience humaine que Hobbes avait subordonné à la politique de l'Etat. (...) la morale n'est plus une morale formelle de l'obéissance, elle n'est plus subordonnée à une politique absolutiste, elle affronte les lois de l'Etat (C47-K45).

7. Mais quid de la guerre de succession d'Espagne (1701-1714), de la guerre de succession d'Autriche (1740-1748) ou encore de la guerre de Sept ans (1756-1763)? Le système westphalien n'a nullement la stabilité qu'on lui prête : le mode dynastique de dévolution du pouvoir en régime hiérocratique (et non démocratique) est un facteur belligène chronique en Europe même. 
Typique du premier Koselleck, cette lecture représente un véritable forçage du texte, afin d'accréditer la thèse générale de KuK sur l'époque. En ne hiérarchisant pas les trois types de lois (divine, civile, philosophique), Locke aurait ainsi préprogrammé un conflit général des normativités ${ }^{8}$. Il n’y a pourtant chez Locke ni conflit ni absence de hiérarchie. Les «lois civiles» fixent des principes d'action infranchissables sous peine de sanction; elles cadrent le licite quant à la vie, la «liberté» et la «propriété privée». Les «lois divines» le font également à un niveau plus intérieur de la conscience religieuse affrontée à la question des fins dernières. Les jugements moraux ordinaires et le contrôle social au sein de la société civile parachèvent - au niveau des mœurs - ce bornage des pensées et des actions individuelles. Malgré cela, Koselleck ne veut voir chez Locke que l'annonce du divorce entre la loi positive de l' Etat et loi philosophique de la société civile.

Comme en plusieurs passages de KuK, le défaut de méthode est quadriple au regard des conclusions générales : Koselleck ne s'appuie le plus souvent que sur un corpus philosophique, et qui plus est sur fort peu d'auteurs; du corpus de ces rares auteurs, il ne retient que très peu de textes; il les sursollicite très fréquemment pour le besoin de sa démonstration; enfin, après vérification, certaines citations et références cruciales pour le propos se sont avérées introuvables dans les éditions citées. Quatre biais pour le moins dirimant au regard du caractère massif des thèses critiques soutenues quant aux Lumières. C'est pourquoi il me semble qu'on peut à bon droit parler d'un "premier Koselleck à thèse», davantage situé du côté d'une certaine théorie politique normative, néo-hobbésienne, que d'une pratique méthodiquement réglée de l' histoire.

Dans ce chapitre 2 de KuK, la démonstration de Koselleck consiste «in fine» à aligner toutes les planètes. Le «for intérieur» du sujet de l'absolutisme passe de l'espace privé vers l'espace public. Les convictions «religieuses» deviennent des articles de foi «moraux» «sécularisés». La critique philosophique se pense comme mieux sachante et mieux disante moralement ; elle devient la pratique généralisée d'un groupe social (Philosophes, cafés, salons, clubs, loges maçonniques) se pensant comme cette élite qui doit impulser les transformations générales de la société future. La «République des lettres» prétend détenir l'imperium véritable et s'adonne à une guerre illimitée de tous contre tous. Héritière de Pierre Bayle, la «critique» est sanctifiée par Voltaire comme la «10 Muse», ouvrant ainsi la voie à Diderot, à Beaumarchais et à son Figaro, jusqu'à la Révolution française.

C’est l' heure de ce que Koselleck appelle le «Règne de la Critique». La formule figure en français dans le texte allemand (KuK 51), avec la double majuscule, sans guillemets ni référence à aucune source. Elle concentre la thèse générale du livre, à savoir l'inversion fondamentale de l'ordre de fondation de la société politique depuis le De cive de Hobbes : l' imperium est passé du côté de la libertas, la liberté instituée du côté de la licence pré-politique.

8. «Du fait qu'il n'a pas déterminé le contenu des lois morales mais ne les a décrites que formellement dans leur genèse spécifique, il pense qu'elles pourraient coïncider dans leur contenu concret avec les lois politiques ou divines ; c'est pourquoi il a pu laisser coexister tranquillement les différents pouvoirs. Le fait qu' il ne les a pas ressentis comme antinomiques est un trait de l'originalité de sa théorie politique » (C48-K48). 
C’est désormais la société, et la puissance critique de la philosophie, qui est en passe d'usurper le trône et de créer les conditions d'un nouvel état de nature pré-stasique.

Selon Koselleck, ce sont les clubs ainsi que les différentes diasporas protestantes (notamment en Hollande et en Angleterre) qui vont être les relais sociologiques concrets susceptibles de faire infuser dans la société les axiomes comme l'ethos de la Critique. En France, le Club de l'Entresol de l'abbé D’Alary et de l'abbé de Saint-Pierre déchante toutefois de ne pas avoir débattu à huis clos, dans la confidence et le «secret». Le cardinal Fleury le contraint de fermer en 1731. Les loges maçonniques se mettent ultérieurement à l'abri de ce genre de mésaventures en généralisant la pratique du secret. Elles y ajoutent la leçon de «l'égalité» transclasse dans la fraternité des loges (à l'instar de l'égalité entre hommes et femmes dans les «salons»). C'est pour Koselleck un avant-goût de la «liberté civile», comme le prélude pratique aux principes philosophiques à réaliser: «La liberté en secret devient le secret de la liberté »(C62-K60). Koselleck affectionne ce genre de renversement de formule valant démonstration. Dans les loges maçonniques, les individus s'éprouvent alors en tant qu'égaux, fraternels, libres dans le secret, et représentants d'une moralité et destinée supérieure de l'humanité. Ces petites communautés morales se rêvent en lieu et place des Etats absolutistes, immoraux et arbitraires, à l'instar de la raison d'Etat. C'est l'horizon du cosmopolitisme moral (chez Lessing, mais aussi Weishaupt, Knigge et Wieland) qui se substitue à la réflexion sur les conditions de possibilité minimales de tout ordre politique pacifié en interne comme en externe.

Toutes ces aspirations se heurtent pourtant à la structure politique de l'absolutisme qui dénie aux sujets toute participation et responsabilité politiques. Le blocage politique est complet; les couches sociales montantes n'ont d'autre alternative que de sur-investir l'inflation verbale critique puis la crise concrète de l'Etat absolutiste. L'analyse de Koselleck est ici très franco-centrée; il ne tient nullement compte de la diversité des systèmes absolutistes et des répertoires de contestation induits. Il entend néanmoins repérer une montée des langages dualistes, agonistiques, du politique (C104-K102). C'est à cette occasion qu'il jette les premières bases de sa sémantique historique autour des grandes notions antithétiques alors débattues (Gegenbegriffe, C84-K83) . Cette structuration politique et discursive est explosive ; la Critique est achevée, la Crise peut bientôt commencée. Le parti des Jacobins n'aura qu'à se baisser pour ramasser l'imperium arraché par la société civile des mains de l'Etat.

La «surcharge schmittienne» du chapitre 1 de KuK est redoublée dans le chapitre 2 d'une «surchage cochinienne», par référence aux travaux d'Augustin Cochin sur les loges maçonniques et le jacobinisme (C92-K91). Du chapitre 1 au chapitre 2, la démonstration boucle sur elle-même. Logée dans le for intérieur du sujet absolutiste, la réserve destituante de «droit naturel» s'est muée en potestas indirecta de la société civile contre l’Etat: « La morale est le souverain présomptif »(C70-K68; C125-K124).

9. Cf. les longues notes de bas de page de l'édition 1959, rejetées en annexes séparées dans la traduction française de 1979 (C157 sq.), et complétées dans l'édition définitive Suhrkamp de 1973.

Revista de historiografía 34, 2020, pp. 53-72 


\subsection{Philosophie de l'histoire et futurisation utopique de l'eschatologie}

Ce n'est que dans le troisième et dernier moment de son argumentation que Koselleck aborde la question de la philosophie de l'histoire comme genre réflexif et matrice politique (chapitre 3). C'est là ce que j'appellerai la surcharge löwithienne non seulement de $\mathrm{KuK}$ mais de toute la pointe politique de sa sémantique historique. On sait à quel point Karl Löwith a pour lui compté puisqu' il a traduit Meaning in History (conjointement avec Hanno Kesting) avant de préfacer son autobiographie ${ }^{10}$. Le premier Koselleck de KuK reste largement dans la dépendance de Löwith sur le sujet de la philosophie de l'histoire, son originalité étant de l'articuler à la proposition schmittienne sur la structure politique de l'Etat absolutiste. Ce n'est que par la suite, dans la discussion avec Hans Blumenberg en 1975, que Koselleck actera la nécessité de se démarquer de toute Ableitungsthese stricte faisant de la philosophie de l'histoire une version sécularisée de la théologie ; il accordera alors qu'il serait plus pertinent de n'en retenir que la dynamique métaphorique, au niveau des mutations du langage socio-politique moderne ${ }^{11}$.

Pour l' heure, KuK fait du genre naissant de la " philosophie de l' histoire » l' héritière de l'eschatologie chrétienne et le principal discours de dissimulation de la structure conflictuelle réellement à l'œuvre entre la société et l'Etat. «Eschatologie chrétienne sous sa forme modifiée de progrès séculier, éléments gnostiques et manichéens dissous dans le dualisme de la morale et de la politique, cyclologies antiques, enfin récente légalité scientifique appliquée à l' histoire, tout cela a contribué à sa naissance» (C110-K108). Interdits en 1785, les Illuminati franc-maçons de Bavière «font du plan de salut de Dieu, en le sécularisant, un plan rationnel de l' histoire» (C112). «Grâce à cette identification du plan indirectement politique avec le cours de l' histoire, on dissimule la possibilité de la révolution tout en la provoquant» (C113). La décision ultime, l' issue de la Crise, est ajournée mais par là même déclenchée: « La tension entre l'Etat et la société se décharge apparemment dans l'avenir lointain » $(\mathrm{C} 113)^{12}$.

Le point nodal est qu'en couplant morale et philosophie de l' histoire les Illuminati abolissent les conditions quasi transcendantales du politique: « Haut et bas, intérieur et extérieur cessent d'être des phénomènes historiques, car avec l'épanouissement de la morale disparait toute autorité et donc aussi l' Etat »(C112-K110). En futurisant le progrès moral ainsi que la réconciliation de l' humanité, les franc-maçons ruinent la variante hobbésienne de l'autorisation absolutiste. C'est la conscience individuelle (et non l' auctoritas) qui est désormais la source du droit; la légitimité morale prime sur la légitimité absolutiste. Le pouvoir «doit devenir une fonction

10. R. Koselleck, «Dankrede»... op. cit., 45.

11. Cf. l'excellente analyse de Gennaro Imbriano, Der Begriff der Politik. Die Moderne als Krisenzeit im Werk von Reinhart Koselleck, Francfort/Main, 2018, 120 s.

12. Je me réfère à la version de 1959 traduite en français en 1979 car la version remaniée allemande de KuK en 1973 a été sur ces points largement modifiée par Koselleck (K110-113). Mon hypothèse - provisoire - est que Koselleck avait entretemps, grâce aux articles du Lexikon, considérablement élargi sa documentation sémantique sur le concept de «Geschichte» et la «Verzeitlichung » de sorte qu'il a procédé à la révision du texte de 1959. 
de la société», de même que doit advenir la «moralisation de la politique» (C123-K123). Mais l'argumentation koselleckienne va encore au-delà; elle consiste à affirmer que c'est en dissimulant aux acteurs d'alors le processus de re-moralisation/destruction en cours du politique que la philosophie de l' histoire a ouvert la voie à la mise en crise effective du régime absolutiste. «Voiler cette dissimulation en tant que telle, c'est la fonction historique de la philosophie bourgeoise de l' histoire» (C132-K133; C155-K157).

Dans un passage entre tous fameux du livre III de L'Emile, Rousseau se fait l'écho de la tension alors palpable: « Nous approchons de l'état de crise et du siècle des révolutions » (cité C133-K133). Il est identifié par Koselleck comme un des points de basculement du «Règne de la Critique» futurisée vers le cycle totalitaire moderne : «Rousseau cherchait l'unité de la morale et de la politique ; il a trouvé l'Etat total, la révolution permanente cachée sous le manteau de la légalité »(C135-K136). «La dictature se distingue de l'absolutisme dans la mesure où elle veut intégrer l'intérieur du particulier que Hobbes avait retranché de l'Etat» (C137-K137). «C'est la tâche du chef de créer l'identité fictive de la morale et de la politique. (...) Il faut guider, non seulement les actions, mais surtout les convictions. (...) La terreur est son chemin et l'idéologie son mode» (C138-K138). La charge est pour le moins massive. C'est dans les passages sur Rousseau que Koselleck porte à son comble sa rétroprojection des catégories politiques de la guerre froide.

L'ultime point de la reconstruction historique de KuK consiste à montrer comment, avec l'Abbé Raynal ${ }^{13}$, la tension verticale entre société (critique) et Etat (absolutisme) minant l'ancien monde européen est recodée horizontalement dans une sorte de spatialisation de l'utopie futurisée. C’est ce que Koselleck appelle la «différence atlantique», soit le «masque géographique» du premier masque dissimulant de la philosophie de l' histoire. Ce masque de second degré, ce sont les colonies américaines, cette anticipation de l'humanité réconciliée par-delà l'ordre politique des séparations. La vérité morale (celle portée par la société, et non l'Etat) adviendra dans le Nouveau Monde au prix d'une coupure politique et géographique d'avec la vieille Europe. Koselleck résume le propos en ces termes : « Raynal fait basculer de sa verticalité sociale la différence entre l’ancien régime et la nouvelle société et lui donne un masque géographique. Le dualisme moral qui jusqu'ici guidait la critique se dilate jusqu’à la différence atlantique, laquelle devient le signal historique de la crise qui entraîne un renversement définitif» (C149-K150). Ici encore, après vérification des passages invoqués, on peut dire que Koselleck sur-sollicite le texte de Raynal qui ne dit nulle part cette montée de la guerre civile sur fond de philosophie de l' histoire moralisante; à propos de la résistance passive des colonies américaines au timbre, Raynal précise en un sens tout différent qu'il n'y a pas eu de guerre civile alors qu'on aurait pu s'attendre à ce que ce soit le cas en un siècle de fanatisme ${ }^{14}$. Là où Raynal n'aurait fait que prophétiser la «différence atlantique», Thomas Paine - avec sa revue The Crisis (1776-1783) - contribue à faire advenir la décision ultime dans les Treize Co-

13. G.-Th. Raynal, Histoire philosophique et politique des établissemens \& du commerce des européens dans les deux Indes, 6 vol., s.n., Amsterdam, 1770.

14. G.-Th. Raynal, Histoire..., op. cit., VI, 410. 
lonies d'Amérique. Et l'auteur de KuK de scruter le bouclage cybernétique de ce militantisme en montrant à grand peine comment Raynal, dans les rééditions successives de son ouvrage, cite les textes de Paine parus entretemps sur ces questions névralgiques de guerre civile et de justice utopique réalisée par le Nouveau Monde (C152-K152).

La boucle de KuK se referme ici. La «Critique» philosophique avait été l' hypocrisie de premier degré donnant l'assaut contre l' Etat absolutiste qui l'avait pourtant rendue possible. La «philosophie de l' histoire» sera «l' hypocrisie de l' hypocrisie» (C155-K157), soit cette hypocrisie de second degré dissimulant la première sous la forme temporalisée de l'utopie d'abord et du «masque» spatial de la «différence atlantique» ensuite. Les conclusions de KuK sont dès lors claires au seuil de 1789. Elles pourraient être aisément résumées en reformulant comme suit la maxime hobbésienne princeps: Moralitas (ut Critica ut Utopia ut Historia) non auctoritas facit legem. Remède à la stasis, surplombant son monde, le Léviathan armé du glaive et de la crosse épiscopale ne repose plus sur la terre ferme, et le lien généalogique direct avec le $\mathrm{XX}^{\mathrm{e}}$ siècle est clairement affirmé. «De la critique souveraine naît la souveraineté de la société» (C154-K155). «L'utopie comme réponse à l'absolutisme ouvre ainsi le procès des temps modernes. (...) La guerre civile, sous la loi de laquelle nous vivons encore aujourd' hui» (C155-K156), voilà la contribution au monde moderne de la « souveraineté de l' utopie » (C156-K157) et des « militants bourgeois » en mal de participation par la «moralisation de la politique » (C155-K156). L'utopie, ce chèque sans provision sur l'avenir, est une impasse: «La traite a été présentée pour la première fois en 1789 » (C156-K157). Pour Hanno Kesting, Roman Schnur, Nikolaus Sombart et Reinhart Koselleck, ces jeunes étudiants de Heidelberg obnibulés par la " guerre civile mondiale » (Weltbürgerkrieg) au début des années $1950^{15}$, cette traite continuait d’être présentée au monde moderne selon des variantes toutes aussi coûteuses les unes que les autres en désastres humains alors même que chacune était induite par une seule et même structure idéologique dualiste, autorisant la montée aux extrêmes depuis l'ouverture du cycle révolutionnaire utopique à la fin du XVIII siècle. Le face à face à la fois idéologique et thermo-nucléaire des USA et de l'URSS durant la guerre froide ne représente pour Koselleck que le dernier avatar de cette structure agonistique. C'était «l'ère du délai» et le «temps de la fin», selon Günther Anders, à savoir : non plus «le royaume sans l’apocalypse» via l'utopie mais «l'apocalypse sans le royaume» ${ }^{16}$. L'inquiétude était partagée, générationnelle, car décuplée par la puissance mortifère de la technique. Si elle s'est déplacée depuis en direction de la collapsologie climatique, cela ne saurait faire illusion sur le fait que la menace thermo-nucléaire demeure aujourd' hui encore parfaitement possible.

\section{Les avatars de la Crise: Sattelzeit, temporalisation et accélération}

Le travail d' habilitation sur la Prusse a considérablement déplacé le questionnaire koselleckien en direction de l'histoire des concepts et des temporalités, articulées à une histoire so-

15. N. Olsen, History in the plural: an introduction to the work of Reinhart Koselleck, New York, 2012. 16. G. Anders, Endzeit und Zeitenende: Gedanken über die atomare Situation, Münich, 1972. 
ciale exigeante mais in fine subordonnée à la réflexivité interprétative ex post de l' historien ${ }^{17}$. C'est à partir de ce grand déplacement que Koselleck va reprendre et reformuler, au travers du

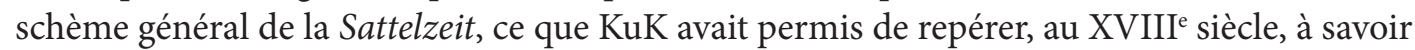
la montée du genre de la «philosophie de l'histoire» ainsi qu'un processus de sécularisation intra-mondain des agendas critiques de justice socio-politique. Cette grande thématique se trouve donc reprise mais à la faveur d'inflexions propres au nouveau champ d'investigation du langage socio-politique allemand moderne, en ses « concepts fondamentaux ${ }^{18}$. Rédigée pour partie dès l'automne 1963, publiée selon une première version en $1967^{19}$, l' introduction générale (1972) au grand dictionnaire des Geschichtliche Grundbegriffe fixe durablement le programme de la Begriffsgeschichte et le questionnaire englobant de la Sattelzeit. L'évolution de ce texte entre 1963/67 et 1972 n'a jamais été analysée de près; scrutons en les inflexions principales quant à la thématique de la crise et de la grande transformation moderne.

\subsection{La Sattelzeit (1963-1972)}

Le mot de «Sattel-zeit» (puis «Sattelzeit») apparaît pour la première fois en 1963/6720 ; il est ordonné à une « hypothèse heuristique » déjà bien stabilisée, et équivalente au texte remanié de $1972^{21}$. C'est le « début du monde moderne»(Beginn der Neuzeit) ${ }^{22}$ qu'il s'agit d'identi-

17. R. Koselleck, Preußen zwischen Reform und Revolution. Allgemeines Landrecht, Verwaltung und soziale Bewegung von 1791 bis 1848, [1965] Stuttgart, 1967; $2^{e}$ édition révisée, Stuttgart, 1975.

18. Pour une présentation générale de la Sattelzeit, cf. J. Stefan, "Die Sattelzeit. Transformation des Denkens oder revolutionärer Paradigmenwechsel? », in A. Landwehr (ed.), Frühe Neue Zeiten. Zeitwissen zwischen Reformation und Revolution, Bielefeld, 2012, 373-388.

19. R. Koselleck, "Richtlinien für das 'Lexikon Politisch-sozialer Begriffe der Neuzeit' ", in Archiv für Begriffsgeschichte, vol. 11, 1967, Bonn, 81-99.

20. R. Koselleck, «Richtlinien... », op. cit., 82, 91 et 95 (pour « Sattel-zeit ») et 96 et 99 (pour « Sattelzeit ») ; comparer à la version de 1972, in Koselleck, «Einleitung », in Otto Brunner, Werner Conze et Reinhart Koselleck dir., Geschichtliche Grundbegriffe : historisches Lexikon zur politisch-sozialen Sprache in Deutschland, vol. 1, Stuttgart, Klett-Cotta, 1972, XIII-XXVII, ici XV et XXVI (texte désormais cité par l'abréviation «1972, GG, I »).

21. R. Koselleck, «Richtlinien...» op. cit., 81-82: «Es geht darum, den Begriffswandel aus vorrevolutionärer Zeit über die revolutionären Ereignisse und Wand-/lungen hinweg bis in unseren Sprachraum hinein zu verfolgen. Das heuristische Prinzip dabei ist, daß bei gleichen Worten erst seit der Mitte des vorigen Jahrhunderts der heutige Bedeutungsgehalt soweit feststeht, daß er keiner 'Übersetzung' mehr bedarf. Der heuristische Vorgriff führt sozusagen eine 'Sattel-zeit' ein, in der sich die Herkunft zu unserer Präsenz wandelt. Begriffe dieser Zeit tragen ein Janusgesicht : rückwärtsgewandt meinen sie soziale und politische Sachverhalte, die uns ohne Übersetzung und Deutung der Worte nicht mehr verständlich sind, vorwärts und uns zugewandt haben sie Bedeutungen gewonnen, die einer Übersetzung nicht mehr bedürftig sind. Begrifflichkeit und Begreifbarkeit fallen seitdem zusammen » (comparer mot pour mot à 1972, GG, I, XV).

22. R. Koselleck, « Richtlinien... », op. cit., 91, également 84 (comparer à 1972, GG, I, XV pour « Beginn der 'Neuzeit' ", XIX pour «Entstehung der Neuzeit » ainsi que "Umwandlungsprozeß zur Moderne » et XXIV pour « Strukturwandel der Geschichte»). 
fier, au fil de ses mutations conceptuelles qui seraient les indices de changements tour à tour politiques, socio-économiques et expérientiels. Une double révolution, politique et industrielle, engage alors une transformation profonde et rapide du monde hiérarchique hérité des statuts, " ordres » et " états » (Stände) ${ }^{23}$. C'est ainsi que se serait durablement ouverte la grande alternative dynamique de la «Tradition » et de la "Révolution », jusqu'à l'orée de notre «présent » politique ${ }^{24}$. Le dispositif interprétatif de 1963/67 est limpide. Le passé de la société d'ordres se trouve durablement reconfiguré durant de la Sattelzeit (1750-1850), via l'agonistique sémantique de la «Tradition » et de la «Révolution»: et cela, jusqu'à ce que se stabilisent les cadres conceptuels de la société présente - depuis pour nous immédiatement intelligible, sans que soit requis un travail profond d'interprétation/traduction conceptuelle.

Cette première version de la Sattel-zeit comporte tout ce sur quoi Koselleck reviendra par la suite. Ceux qu' il appelle encore des Zentralbegriffe ${ }^{25}$ deviendront en 1972 des Grundbegriffe, à savoir non pas de simples mots (Wörter), mais des notions socio-politiques plurivoques, condensant en elles des enjeux sociétaux si polysémiques qu'ils demeurent investissables par de nombreux groupes sociaux et porte-paroles antagoniques. La dimension non pas seulement «sémantique» mais pragmatique des jeux de discours (en situation, dans des contextes spécifiques), cette dimension essentielle non idéaliste est d'emblée posée au travers de la question cardinale du Cui bono? Autrement dit, à qui s'adresse telle ou telle prise de parole? En provenance de qui, de quel groupe social ? Selon quelles notions inclusives ou bien exclusives, voire stigmatisantes? Selon quels intérêts économiques et quelles visées tactiques? A quel niveau infrastructurel précis - spatial et médiatique - de l'espace public du moment, selon quels effets et rayonnement spécifiques, etc. ?26 Tous ces réquisits de contextualisation des discours sont posés dès 1963/67, et seront réitérés comme tels chroniquement par la suite, en dépit des lectures tronquées et délibérément polémiques de certains - faute de pouvoir se nourrir méthodologiquement d'autre chose que de caricatures anti-koselleckiennes ${ }^{27}$.

23. La notion de "Wandel » (ailleurs « Umbruch ») est récurrente, cf. R. Koselleck, « Richtlinien... », op. cit., 82, 84, 86, 91, 99.

24. R. Koselleck, « Richtlinien... », op. cit., 91 et 99.

25. R. Koselleck, «Richtlinien... », op. cit., 89.

26. R. Koselleck, «Richtlinien...» op. cit., 87-88 (comparer à 1972 GG, I, XX). La question des « sources » discursives mobilisables (Quellen) recoupe cette question du « cui bono » pragmatique, et Koselleck distingue d'emblée entre un niveau " moyen ", " haut " et " bas " relativement à l'échelle matérielle concrète et littéraire-philosophante (ou pas) de l'espace public (op. cit., 97-98, à comparer à 1972, GG, I, XXIV-XXV).

27. Partiel autant que partial, caricature mal informée autant que désinformante, le dialogue de sourds avec Quentin Skinner et John Pocock est à cet égard désarmant, autant que stratégiquement révélateur. C’est sans doute parce que toute cette tradition de recherche, et leurs légions d'épigones contemporains académiquement employables, n'a jamais eu qu'un rapport tout littéraire, d'érudition intertextuelle, aux structures d'action du politique, de l'économique, du social et du religieux. 
Mais, plus fondamentalement, les quatre questions heuristiques de l' hypothèse générale de la Sattelzeit ne sont encore qu'esquissées ${ }^{28}$. En 1972, en effet, Koselleck avance quatre clefs de lecture possibles du passage de l'ancien régime au monde moderne. Premièrement, un mouvement de «démocratisation» (Demokratisierung) des concepts socio-politiques ${ }^{29}$, soit donc le fait que le langage politique en soit venu à inclure beaucoup plus d'acteurs et de réalités sociales que les vieilles catégories de la société d'ordres (Stände) ne permettaient d'en impliquer. Dans le programme de 1963/67, Koselleck ne saisit ce premier grand processus de transformation sémantique qu'au travers de l'agonistique centrale « Tradition »/ « Révolution » ${ }^{30}$.

Le second grand processus de changement pointé en 1972 relève de la catégorie de «temporalisation» (Verzeitlichung) ${ }^{31}$, soit donc l'idée que les grandes catégories modernes du politique intègreraient désormais des éléments de philosophie de l'histoire allant bien au-delà des langages passés de la domination socio-politique spatialisée - e.g. «Emanzipation» versus «Herrschaft». Ce processus-là n'est encore qu'effleuré en 1963/67 par des remarques adventices sur «l'accélération» (Beschleunigung) et l'apparition de concepts perspectivistes temporalisés partisans (parteigebundene, geschichtsphilosophische Perspektivbegriffe) ${ }^{32}$. Par rapport à KuK, cela constitue néanmoins un déplacement notable en direction des sémantiques temporalisées modernes. La double thématique de la temporalisation et de l'accélération commence à prendre la place de sa première critique, moins sémantique que doctrinale, voire idéologique, de la «Kritik» moderne en tant que factrice de « Krise » de l'Etat absolutiste pacificateur.

La troisième série de transformation sémantique avancée en 1972 souligne le degré croissant d' «idéologisation» des langages socio-politiques modernes (Ideologisierbarkeit) ${ }^{33}$. C'est sur ce point où la continuité est la plus forte avec le pré-programme de 1963/67. Dans un cas comme dans l'autre, Koselleck souligne le "degré d'abstraction » montant des sémantiques à l'œuvre, leur caractère de plus en plus évidé, aveugle aux concrétudes expérientielles, ainsi que leur caractère manipulable ${ }^{34}$. Déjà au cœur de KuK, le soupçon d'inflation sémantique, crisogène, de la modernité est ici encore très fort mais désormais rapporté à la

28. La formule-clef ramassant les différents processus aperçus en 1963/67 est la suivante : « Die Begriffe sind also nach dem jeweiligen Erfahrungshorizont aufzuschlüsseln : inwieweit dieser 'geschichtsphilosophisch', 'ideologisch', 'abstrakt' ist, kann nur die Einzelanalyse ergeben » (Koselleck, "Richtlinien »... op. cit., 93). Le résumé symétrique, stabilisé, de 1972 s'énonce ainsi : « Alle genannten Kriterien, die Demokratisierung, die Verzeitlichung, die Ideologisierbarkeit und die Politisierung bleiben unter sich aufeinander verwiesen. Ohne jeden Anspruch auf Vollständigkeit behalten sie heuristischen Charakter, um den Gebrauch neuzeitlicher Terminologie gegen deren vorrevolutionären Zusammenhänge abgrenzbar zu machen. Aus dem heuristischen Vorgriff folgt nun keineswegs, daß ihn die Geschichte jedes Begriffs bestätigen müßte » (GG, I, XVIII).

29. R. Koselleck, « Einleitung », op. cit., XVI.

30. R. Koselleck, « Richtlinien... », op. cit., 91 et 99.

31. R. Koselleck, « Einleitung », op. cit., XVI-XVII.

32. R. Koselleck, « Richtlinien... », op. cit., 88, 91, 92 et 93.

33. R. Koselleck, « Einleitung », op. cit., I, XVII-XVIII.

34. R. Koselleck, « Richtlinien », op. cit., 91 ( Ideologisierung »), 92 (« Abstraktionsgrad »; " Manipulierbarkeit ») et 93 (« abstrakt»).

Revista de historiografía 34, 2020, pp. 53-72 
morphologie même (infra-sémantique) des langages politiques modernes. Et c'est justement la force de l'argument koselleckien que de porter également sur la «forme», et non uniquement sur le «contenu » signifiant des discours. Ce que l'introduction générale au Lexikon de 1972 ajoute à cet argument de 1963/67, c'est la notion de «singuliers collectifs» (Kollektivsingulare), soit donc l'idée que des concepts processuels existant auparavant au pluriel auraient été de plus en plus mobilisés au singulier majusculé (le Progrès, l'Histoire, la Liberté, etc.) ${ }^{35}$.

La quatrième et dernière transformation englobante énoncée en 1972 est celle d'une «politisation» (Politisierung) sémantique généralisée, à savoir l'idée d'une augmentation drastique, lors du passage à la modernité, des modes de désignation polémiques, polarisés, non seulement des enjeux mais des positions groupales (soi/ les siens versus les autres) au travers de mots-slogans - e.g. "Aristocrates» versus «Démocrates», «Réactionnaires» versus «Révolutionnaires», etc. ${ }^{36}$. On retrouve ici la question des «dualismes» et des Gegenbegriffe (concepts antagoniques, relationnels) omniprésente dans KuK (jusqu'au sous-titre du tapuscrit de 1954) ; elle n'est toutefois plus circonscrite aux discours des Lumières, des loges-maçonniques et des philosophes de l' histoire ; elle devient une des grandes matrices interprétatives de la morpho-sémantique moderne. «Politisation» agonistique partout donc en 1972. Le pré-programme de 1963/67 thématise déjà le point, mais moyennant la notion de Polemisierung (et de Gegenbegriffe $)^{37}$. Cette notion intermédiaire ne change toutefois rien à la forte continuité de la visée heuristique et du diagnostic.

\subsection{Temporalisation et accélération : une sur-accentuation durable et problématique}

Après le lancement du Lexikon en 1972, on peut repérer une ultime inflexion durable de la pensée de Koselleck, à savoir une sur-accentuation manifeste de la thématique de la «temporalisation» et de l' «accélération». Cela permet à Koselleck de maintenir une double thèse: 1) tout d'abord, celle de la Sattelzeit en tant que grand basculement moderne (révolution politique, révolution industrielle et mutations des vieux répertoires métaphoriques de l'attente hérités de l'apocalyptique chrétienne) ; 2) ensuite, la thèse d'une accélération matérielle inédite de la modernité.

Cette seconde proposition se décline en deux arguments distincts, et cela jusqu'aux derniers textes et interventions publiques de Koselleck. D'une part, il y a l'idée que l'accélération matérielle moderne pousse jusqu'à son point de possible rupture la gouvernabilité politique des sociétés à l' heure de la mondialisation économique et de la crise climatique globale (à l' heure de l'anthropocène ou du capitalocène dirions-nous aujourd' hui). D'autre part, il y a l'idée d'une synchronicité ubiquitaire inédite des images et des affects politiques à l'heure du village global médiatique; c'est ce que Koselleck appelle la convergence de «l'image » et

35. R. Koselleck, «Einleitung », op. cit., I, XVII.

36. R. Koselleck, «Einleitung», op. cit., I, XVIII.

37. R. Koselleck, « Richtlinien... », op. cit., 88 et 92. 
de «l'événement » depuis 2001, suite au second crash (sur la seconde tour) du World Trade Center le 11 septembre ${ }^{38}$. Il s'agit bien évidemment ici d'une variation sur le thème méta-politique fondateur de KuK: soit le diagnostic d'une opinion publique internationale en tant que scène globale de nouvelles « justes causes » (i.e. le djihadisme contemporain), remoralisant l'espace neutre du politique et enclenchant un nouveau cycle, désormais transnational, de « guerre civile» (stasis). Depuis KuK, les thèses fondamentales de Koselleck sur la modernité ont tour à tour été reformulées autour de quelques gros noyaux sémantiques aisément identifiables: Kritik, Geschichtsphilosophie, Krise, Bürgerkrieg, Revolution, Geschichte schlechthin, Verzeitlichung et Beschleunigung. C'est cette sur-accentuation ultime de l'une des quatre matrices interprétatives de «l'époque-charnière » (1750-1850), à savoir le processus multicausal (matériel/sémantique) de «temporalisation »/« accélération » qui est à mon sens le ressort - infiniment ductile - de la grande fortune de Koselleck jusqu’à nous.

Bien des choses continuent toutefois de paraître problématique dans la notion de Sattelzeit. Premièrement, affirmer à l'instar de Koselleck que nos langages socio-politiques sont pour l'essentiel homogènes à ceux qui se seraient stabilisés vers 1750-1850 au travers d'un quadruple processus de "démocratisation », «temporalisation », « idéologisation " et " politisation ", une telle affirmation ne peut que porter au scepticisme. Entendons par là qu'elle nous empêche de penser ce qui a pu radicalement changer depuis en termes de processus englobants du politique (processus tour à tour sémantiques, pragmatiques, institutionnels et matériels de tous ordres) ${ }^{39}$. En second lieu, les propositions initiales, fortes, de Koselleck fondant «l'histoire des concepts » conduisent à sur-estimer la dynamique propre, factrice d'histoire, des langages socio-politiques. Il y a là une sur-détermination causale à laquelle il convient de résister. J'y vois pour ma part de surcroît une contradiction interne à la pensée de Koselleck lui-même, qui a par ailleurs continûment affirmé que l'effectivité historique ne se résorbait nullement dans le seul langage des acteurs mais renvoyait toujours à des structures anté-prédicatives ainsi qu'à des conditions de possibilité extra-langagières ${ }^{40}$.

Mais surtout - en troisième et dernier lieu -, c'est la sur-accentuation du schème interprétatif de la «temporalisation »/ «accélération » qui pose problème. A lui seul, il ne saurait suffire à penser la modernité dans ce qui fait sa spécificité normative, factrice d'histoire, à savoir: le long cycle de démocratisation qui s'est ouvert à partir de la révolution américaine

38. R. Koselleck et C. Dutt, " Geschichte(n) und Historik", Internationale Zeitschrift für Philosophie, 2/2001, 257-271, repris in Id., Erfahrene Geschichte. Zwei Gespräche, Heidelberg, 2013, 47-67, ici 67.

39. Qu'on me permette de renvoyer aux propositions faites en ce sens, en forme de topique comparatiste, dans la seconde partie de mon étude intitulée : «'Temporalisation' et modernité politique : penser avec Reinhart Koselleck », in Annales H.S.S., 64 année, nº 6, novembre-décembre 2009, 1269-1301, en particulier $1292 \mathrm{sq}$.

40. C'est bien évidemment l'argument-massue contre Gadamer visant à subordonner l'herméneutique (et toute la tradition philologique depuis August Boeckh et le XIX siècle) à la théorie de l' histoire, cf. R. Koselleck, « Théorie de l' histoire et herméneutique » [" Historik und Hermeneutik», 1985], in Id., L'expérience de l'histoire, Paris, 1997, 181-199 ; Id., Zeitschichten. Studien zur Historik, Francfort/Main, 2000, 15.

Revista de historiografía 34, 2020, pp. 53-72 
(1776), française (1789) puis transatlantique ${ }^{41}$. Des plus complexe et prégnant, ce cycle n'est nullement réduisible aux seules mutations sémantiques des concepts fondamentaux ${ }^{42}$, et il ne saurait être secondarisé par le processus de "temporalisation »/ "accélération ». C’est pourtant bien cette secondarisation qui a conduit au re-phrasage contemporain, par la théorie sociologique (H. Rosa), du diagnostic koselleckien de l'accélération jusqu'à en faire un nouveau concept-d'époque, substitut appauvri à la Sattelzeit ${ }^{43}$.

Le problème subséquent à cette inflexion du débat, depuis $\mathrm{H}$. Rosa et sa fortune universelle, n'est pas tellement que les thèses de Koselleck aient été intégralement aspirées par une certaine sociologie. Le problème ne vient pas non plus du fait que ce réinvestissement verbal ait permis de hisser une certaine théorie sociologique au rang de «théorie critique » englobante, prétendant au magistère politique contemporain en même temps qu'à la thématisation de ses remèdes dans le mal (via de la "résonance " tous azimuts, ultime avatar-pharmacon germanique de la Kulturkritik moderne depuis Rousseau) ${ }^{44}$. La difficulté dirimante, à mon sens, vient bien davantage de ce que $\mathrm{H}$. Rosa (et ses épigones) développe sa thèse générale de la modernité comme accélération générale en faisant complètement l'impasse sur les processus proprement politiques et idéologico-sémantiques depuis le XVIII siècle. Il en résulte un grand écart, peu plausible, entre d'un côté des phénomènes d'accélération proprement matériels (mobilisation en capitaux, processus de production, de transports et communication accélérés) et de l'autre des phénomènes trivialement psychologiques, sauf à les ressaisir comme le stade avancé du capitalisme et de l'individuation modernes (baisse de l'attention par hyperconnexion numérique, multi-tasking, burn out et identités situatives liquides).

La démocratie en son sens extensif (régime uniment politique, juridique, social et économique) et la question de ses conditions de stabilisation se sont ainsi évanouis dans le trou noir de la thèse de "l'accélération $»^{45}$; les médiations sémantico-pragmatiques ont été du même coup escamotées, et nous héritons de fait, sans que cela soit dit, de la sur-accentuation

41. L'appréhension de ce long cycle est encore plus complexe si on le fait débuter, comme on le devrait, avec la « révolte des Gueux » néerlandaise contre l’Empire espagnole en 1566.

42. Le processus sémantique de "Demokratisierung " pointé par Koselleck n’épuise, à mon sens, en aucune manière le processus sociétal moderne de "démocratisation ", dont nous sommes bien encore les contemporains, mais des contemporains toujours déçus par construction (relativement aux exigences inhérentes à la promesse même).

43. H. Rosa, "Bewegung und Beharrung. Überlegungen zu einer sozialen Theorie der Beschleunigung ", in Leviathan. Zeitschrift für Sozialwissenschaft, 27e Année, 1999, 386-414; Id., Accélération. Une critique sociale du temps [2005], Paris, 2010; Id., Aliénation et accélération. Vers une théorie critique de la modernité tardive [2010], Paris, 2012.

44. H. Rosa, Résonance. Une sociologie de la relation au monde [2016], Paris, La Découverte, 2018.

45. Ce qui n'est nullement le cas chez un interlocuteur trop peu discuté de H. Rosa, cf. W. E. Scheuerman, «Liberal Democracy and the Empire of Speed », in Polity, vol. 34, n 1, automne 2001, 41-67 ; Id., "Speed, States, and Social Theory: A Response to Hartmut Rosa ", in Constellations. An international journal of critical and democratic theory, vol. 10, $\mathrm{n}^{\circ}$ 1, mars 2003, 42-48; Id., Liberal Democracy and the Social Acceleration of Time, Baltimore, 2004. 
koselleckienne ultime de «l'accélération » mais sans structures politiques, ni sémantiques, ni anthropologiques, et encore moins socio-économiques : bref, nous héritons d'une théorie sociologique à prétention uniment politique et culturelle, écartelée entre matérialités accélérées et résilience (ou non) psychologisante. Soit donc, à la fin des fins, non du tout une éthique de la vertu mais une esthétique de la vie supportable en modernité matériellement accélérée. Avec la théorie du remède dans le mal de l'accélération (la « résonance »), le succès d'estrade est assuré auprès des innombrables individus-atomes entreprenoriaux (ou académiques), numériquement interconnectés et "managés " dans la globalisation. Avec la théorie de la " résonance » - faisant suite à la sur-accentuation koselleckienne de la thématique de « l'accélération ", queue de comète de la Sattelzeit -, nous avons bien affaire à une énième variante d'une micro-politique (en fait un simple perfectionnisme moral) qui a démissionné des leviers macro-structurels de toute grande politique : rien d'autre, finalement, qu'un énième symptôme de crise au sein de nos Etats modernes complexes - jadis stratèges, redistributeurs et maîtres des horloges, mais aujourd' hui toujours plus incapacités par les flux globaux et les acteurs transnationaux.

\section{Conclusion}

Je l'ai indiqué à plusieurs reprises en reconstruisant l'argument de Kritik und Krise; Koselleck déborde largement le genre initialement revendiqué en 1954/59 d'une histoire structurelle de l'ancien régime; il instruit bien davantage le procès généalogique du totalitarisme au $\mathrm{XX}^{\mathrm{e}}$ siècle. Les motivations biographiques et générationnelles sont parfaitement documentées ${ }^{46}$. Ce qui reste cependant tout à fait dans le flou dans $\mathrm{KuK}$, ce sont les critères qui pourraient nous permettre de distinguer entre une critique politique à visée réformatrice légitime et une critique de nature potentiellement totalitaire, en raison de sa structure idéologique, supposément repérable dès la fin du XVIII ${ }^{\mathrm{e}}$ siècle. Le premier Koselleck ne s'embarasse pas de ces chicanes, et passe en cela même complètement à côté de l'essence de la politique moderne : la possibilité et même nécessité de la "critique », démocratiquement instituée, sans la "crise » permanente du régime politique, social et économique. La triple surcharge de son analyse surcharge schmittienne, cochinienne et löwithienne - ne permet pas de tenir ensemble la question des conditions de possibilité pérennes de tout ordre politique (ontologie politique) et celle des critères définitoires d'une légitime critique démocratique de l'ordre des choses tel qu'il va. Il y faudrait une théorie de la paix interne non pas seulement par l'autorisation verticale du Léviathan mais par la justice, c'est-à-dire une théorie (chez lui introuvable) de la loi, du droit et de l'équité. Considéré avec le plus grand sérieux, et loin de toute polémique, son objet d'étude requérait l'analyse du passage de la «structure politique de l'absolutisme» à la «structure politique du démocratisme» - rien de moins que notre ordre politique à continûment stabiliser depuis. Koselleck n'est resté qu'au seuil du problème. Certes, il a très tôt repé-

46. Cf. N. Olsen, History... op. cit., ainsi que Gennaro Imbriano, Der Begriff... op. cit. 
ré la structure contradictoire interne de la pacification verticale absolutiste, mais il est demeuré dans la critique négative de certains discours d'opposition, et cela en raison même de ses prémisses philosophiques d'alors : le Léviathan comme katechon et seul remède à la stasis pré-politique. Bref, il a choisi de ne raconter qu'une partie de cette histoire et de ne traiter, philosophiquement, que la part la plus triviale de la question : l'utopisme moralisant futurisé en lieu et place d'une théorie moderne de la démocratie politique, sociale et économique.

Comme Jacob Talmon, Leo Strauss, Eric Voegelin et tant d'autres, Koselleck n'est en cela que le fils de son temps (le totalitarisme et la guerre froide) et de son environnement intellectuel. Kritik und Krise ne serait-il donc qu' un simple document, fascinant, d' histoire contemporaine ? Pour partie, assurément. Les thèses inaugurales de Koselleck ressortissent à un intérêt de connaissance propre aux expériences de la mort collective violente au XX siècle (à l'inclusion des " expériences primaires » de Koselleck lui-même au sortir de la Seconde Guerre mondiale $)^{47}$. De Kritik und Krise à l' " iconologie politique » de la mort violente, c'est sans doute une force de ses hypothèses interprétatives que d'avoir tenu le fil rouge de la montée aux extrêmes politiques durant la modernité. Mais c'est pour nous aujourd'hui également une de ses faiblesses, à savoir ne pas éclairer dans toute sa complexité, autant dramatique que durablement émancipatrice, la nature profonde du long cycle démocratique moderne. De nature juridico-politique, systémique, sociologique, religieuse, culturelle - et non strictement langagière -, ce cycle démocratique ne peut en effet être réduit à une analyse rétrospective structurale des sémantiques et idéologies totalitaires, qui demeure pourtant tout du long l'implicite existentiel des grandes questions koselleckiennes. Et ceci jusqu'aux différents textes analysant les Feindbegriffe asymétriques, puisqu' ils indiquent à mon sens le moment où Koselleck anthropologise son questionnement initial de KuK sur les «dualismes» et Gegenbegriffe modernes.

Il se pourrait ainsi que son approche de la « crise » ne conserve de pertinence qu'en un sens restreint d' " accélération " matérielle et sociale. En quoi Koselleck anticipait avant la lettre sur les problèmes de l'anthropocène, mais à partir de prémisses philosophiques toutes différentes, en dehors de sa visée propre, par une sorte de continuité et pré-science quasi involontaires. La fortune du «critique de la Crise» ${ }^{48}$ s'élève aussi haut que demeurent imprévisibles les aléas historiques de la ruse.

47. R. Koselleck, « Vielerlei Abschied vom Krieg », in H. L. Arnold, B. Sauzay et R. von Thadden (eds.), Vom Vergessen, Vom Gedenken. Erinnerungen und Erwartungen in Europa. Zum 8. Mai 1945, Göttingen, 1995, 19-25.

48. I. Nagel, « Der Kritiker ... », op. cit. 